\title{
Erratum
}

\section{Analytic Representation for Form Factors and Determination of Radii of Hadrons and Light Nuclei}

Olgierd Dumbrajs and Magdalena Staszel

Z. Physik A280, 383-384 (1977)

We have made a trivial numerical mistake on page 384: $t_{0}=4 m_{\pi}^{2} \approx 1.87 \mathrm{fm}^{-2}$ and not $\approx 0.37 \mathrm{fm}^{-2}$ as stated in the paper. Unfortunately this has led us to a serious mistake of criticising unjustly in the footnote on the same page the fits of the paper by F. Borkowski, G.G. Simon, V.H. Walther and R.D. Wendling, Zeitschrift für Physik A275, 29 (1975). These authors have not used data on $F(t)$ for $|t| \geqq 1.87 \mathrm{fm}^{-2}$ for determining the radius of the proton. Our footnote is wrong.

We regret our unfortunate mistake.

O. Dumbrajs

Institute of Theoretical Physics

Warsaw University

Hoza 69

00-681 Warsaw, Poland 